\title{
Energy transfer in biphasic magnetised interstellar medium
}

\author{
M. Langer and J.-L. Puget \\ Institut d'Astrophysique Spatiale, Université Paris-Sud, 91405 Orsay Cedex, France \\ Received 20 September 2002 / Accepted 6 May 2003

\begin{abstract}
The transfer of energy from large scales to smaller scales is investigated within the context of the neutral atomic phases of the interstellar medium threaded by a magnetic field. More precisely, we derive the reflection and transmission coefficients for linear transverse hydromagnetic waves incident normally on a cloud in slab geometry. Then, we obtain various interesting physical quantities such as the energy density transferred into the cloud. The results obtained differ from those given previously in the literature. We show in particular that the transfer of energy is efficient enough so that $(i)$ the induced internal motions are of the same order of magnitude than the bulk motion of the clouds and (ii) the internal energy density provides significative additional non-thermal support for cloud stability, reaching the same order of magnitude than support by the static magnetic field. Finally, the case of molecular clouds is also briefly considered for which similar results are obtained.
\end{abstract}

Key words. ISM: clouds - waves - ISM: structure - MHD

\section{Introduction}

The Interstellar Medium (ISM) is now understood as a medium in which gravitational instability results from the complex interplay of large scale motions and magnetic fields on the one side and self gravity of the gas on the other side. Furthermore, the ISM is subject to thermal instability and can be described as a polyphasic medium built of a hot ionised phase, a warm medium and a cold medium, all three roughly in thermal pressure equilibrium (e.g. McKee \& Ostriker 1977; Wolfire et al. 1995). Another major constituent of the ISM is made of molecular gas aggregated in large complexes called Giant Molecular Clouds (GMCs). They show an internal hierarchical structure in which no preferred scale emerges (e.g. Pérault et al. 1986; Falgarone \& Phillips 1991). Most of the GMCs are selfgravitating systems and therefore they are not in pressure equilibrium with the other components of the ISM. However, as suggested by results of Falgarone \& Puget (1988), GMCs may constitute by themselves a three phase medium with cold HI, warm $\mathrm{HI}$ and molecular clumps all in approximate thermal pressure equilibrium (McKee 1995).

In the early eighties, Larson (1981) showed that, on the other hand, the dynamics of gravitationaly bound interstellar molecular clouds is dominated by large scale supersonic motions. This also suggested a non linear energy cascade of turbulent type towards small scales (e.g. Scalo 1987). Furthermore, the observation of non thermal velocity dispersions (e.g. Solomon et al. 1987; Caselli \& Myers 1995) in dense regions has confirmed that the ISM is a highly dynamical system. This in turn has led to models of the ISM in

Send offprint requests to: M. Langer,

e-mail: mathieu.langer@ias.u-psud.fr which clouds are transient features formed by turbulent density fluctuations. It has even been argued that thermal instability and thermal pressure equilibrium are irrelevant for the ISM (Ballesteros-Paredes et al. 1999; Vázquez-Semadeni et al. 2000).

Following this, a strong, still ongoing debate emerged, opposing advocates of both models of the ISM, i.e. stable multiphasic on the one hand or dynamic turbulent on the other hand. For long, studies focused either on the role of turbulence or on the development of a multiphasic medium by thermal instability. However, it seems reasonable that, within the framework of the ISM, thermal instability and turbulence are not mutually exclusive. Works considering simultaneously both processes have been done only recently, and their tight interplay is still far from being clear. Indeed, it has been shown that large scale motions (possibly turbulent) may actually trigger condensation by thermal instability (Hennebelle \& Pérault 1999, 2000; Koyama $\&$ Inutsuka 2000). On the other hand, turbulent motions may result as a consequence of the condensation process due to thermal instability itself (Koyama \& Inutsuka 2002; Kritsuk \& Norman 2002). Moreover, depending on the cooling time to dynamical time ratio, condensation by thermal instability may or may not be inhibited by turbulence (e.g. Sánchez-Salcedo et al. 2002).

In any case, it is clear that realistic models of the ISM, including full theory of star formation, require precise understanding of the gravitational and the thermal instabilities in a medium which is naturally subject to large scale motions. The sources of these motions, differential rotation in spiral galaxies and massive stars through supernovæ and expansion of HII regions, make it a non local problem, both spatially and in time. The kinetic energy is stored in these motions and redistributed 
over large distances by the magnetic field. The understanding of this storage and spatial redistribution of the large scale kinetic energy is one of the critical ingredients of a complete theory of the star formation process which is still poorly understood. Furthermore, provided a strong enough magnetic field exists at the time of structure formation, similar physics could be relevant to the case of protogalaxies.

In the context of the magnetised ISM, it now is well known that non-thermal spectroscopic line widths could be due to hydromagnetic waves (Arons \& Max 1975) which sustain internal supersonic (but alfvénic) motions. The waves may carry sufficient energy to oppose external compression or to contribute significantly to cloud support against gravitational collapse. On the other hand, wave-wave interactions may generate density fluctuations in an initially homogeneous medium (e.g. Elmegreen 1999; Falle \& Hartquist 2002).

Hydromagnetic wave contribution to cloud stability has been extensively investigated in the last decades (e.g. Elmegreen 1985; Falgarone \& Puget 1986; Carlberg \& Pudritz 1990; Zweibel \& McKee 1995; Gammie \& Ostriker 1996; Martin et al. 1997; Coker et al. 2000). In this paper, we deal with one specific question which is the efficiency of energy transfer by shear Alfvén waves from orbital motions of clouds, virialised in a larger structure, to internal velocity dispersion of the clouds. We concentrate on the simplified case of slab geometry, reminiscent of the sheet-like and filamentary structure observed in the ISM. We explore the energy transfer in a biphasic medium such as described by models of the ISM (e.g. Field et al. 1969; Wolfire et al. 1995) where the Cold Neutral Medium (CNM) is condensed into clouds embedded in a Warm Neutral Medium (WNM). Unlike recent studies of similar problems (e.g. Coker et al. 2000), we explicitely assume here that the density drops abruptly between the clouds and the intercloud medium, the density contrast between the two media being strong, typically 150 . Such sharp boundaries are the natural consequence of thermal instability which, as we assume here, is not inhibited by turbulent gas stirring (i.e. for cases where the cooling time to dynamical time ratio is less than one - see e.g. Sánchez-Salcedo et al. 2002). Our goal here is not to model self-consistently the complete hierarchy of interstellar clouds where the energy density is roughly scale invariant. By concentrating on the Alfvén wave transfer into a slab cloud delimited by sharp boundaries, we rather aim at demonstrating that the energy cascade from large scales is efficient enough in a magnetised medium subject to thermal instability. First, we derive the exact expressions of the amplitude reflection and transmission coefficients and show that the induced internal velocity perturbation is of the order of the transmitted one (Sect. 2.1). Then, we concentrate on the internal turbulent energy densities and show how internal interferences, which have been overlooked in previous works, between multiply reflected waves, enchance the energy injection. Consequently, externally generated Alfvén waves may contribute significantly to the cloud stability and especially to its pressure equilibrium with the surrounding medium (Sect. 2.2). Finally, restrictions of the formalism developed in this paper and its possible application to molecular clouds are discussed in Sect. 3.

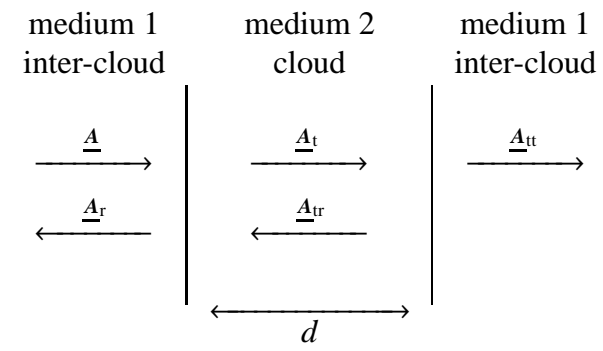

Fig. 1. Schematic overview of the situation. The incident wave $\boldsymbol{A}$ is coming from the left onto a slab of width $d$.

\section{Energy transfer}

We suppose that the ISM is pervaded by a magnetic field $\boldsymbol{B}$ which can be decomposed as follows:

$\boldsymbol{B}=\boldsymbol{B}_{0}+\delta \boldsymbol{B}$

where $\boldsymbol{B}_{0}=\langle\boldsymbol{B}\rangle$ is the slowly varying mean value of the field defining the $x$ direction and $\delta \boldsymbol{B}$ is the wave amplitude transverse to the mean magnetic field. The velocity field can be decomposed in a similar way and we take the mean flow null, so that the only motion $\delta \boldsymbol{v}$ of particles is due to the propagation of shear Alfvén waves.

\subsection{Amplitude reflection, transmission and transfer coefficients}

An Alfvén wave incident on a cloud boundary will be partially reflected back to the inter-cloud medium and partially transferred into the cloud. There, it will propagate up to the opposite boundary where again, a part of it will be reflected whereas the rest will be transmitted into the inter-cloud medium on the other side of the cloud. In a slab geometry (assumed for simplicity), when the steady state is reached (infinite number of reflections inside the cloud), the wave reflection and transmission coefficients are easily calculable, assuming the usual continuity conditions for both the wave amplitudes and their spatial derivatives.

In the inter-cloud medium (medium 1), consider an Alfvén wave $\boldsymbol{A}$ of wave number $k_{1}$ and frequency $\omega$ arriving on the cloud (medium 2) where the wave number is $k_{2}$. Let us call the reflected part of the wave $\boldsymbol{A}_{\mathrm{r}}$, the part transmitted to the other side of the cloud $\boldsymbol{A}_{\mathrm{tt}}$, and the waves inside the cloud $\boldsymbol{A}_{\mathrm{t}}$ and $\boldsymbol{A}_{\mathrm{tr}}$ (transferred, and reflected on the far side respectively cf. Fig. 1).

In the complex notation, and by choosing the phase so that the amplitude of the incident wave is real, we can write the different amplitudes in the following way:

$$
\begin{aligned}
& \underline{\boldsymbol{A}}=\boldsymbol{a} \mathrm{e}^{-\mathrm{i}\left(\omega t-k_{1} x\right)} \\
& \underline{\boldsymbol{A}}_{\mathrm{r}}=\underline{\boldsymbol{a}}_{\mathrm{r}} \mathrm{e}^{-\mathrm{i}\left(\omega t+k_{1} x\right)} \\
& \underline{\boldsymbol{A}}_{\mathrm{t}}=\underline{\boldsymbol{a}}_{\mathrm{t}} \mathrm{e}^{-\mathrm{i}\left(\omega t-k_{2} x\right)} \\
& \underline{\boldsymbol{A}}_{\mathrm{tr}}=\underline{\boldsymbol{a}}_{\mathrm{tr}} \mathrm{e}^{-\mathrm{i}\left(\omega t+k_{2} x\right)} \\
& \underline{\boldsymbol{A}}_{\mathrm{tt}}=\underline{\boldsymbol{a}}_{\mathrm{t}} \mathrm{e}^{-\mathrm{i}\left(\omega t-k_{1} x\right)}
\end{aligned}
$$


where the underlining denotes a complex number. These are the amplitudes of the gas motion. The amplitudes of the velocity are of course obtained by deriving these expressions with respect to time. At the boundaries, we obtain the following continuity relations

$\boldsymbol{a}+\underline{\boldsymbol{a}}_{\mathrm{r}}=\underline{\boldsymbol{a}}_{\mathrm{t}}+\underline{\boldsymbol{a}}_{\mathrm{tr}}$ at $x=0$

$\underline{\boldsymbol{a}_{\mathrm{t}}} \mathrm{e}^{k_{2} d}+\underline{\boldsymbol{a}}_{\mathrm{tr}} \mathrm{e}^{-k_{2} d}=\underline{\boldsymbol{a}}_{\mathrm{t}} \mathrm{e}^{k_{1} d}$ at $x=d$

for the amplitudes (motion) and

$k_{1}\left(\boldsymbol{a}-\underline{\boldsymbol{a}}_{\mathrm{r}}\right)=k_{2}\left(\underline{\boldsymbol{a}}_{\mathrm{t}}-\underline{\boldsymbol{a}}_{\mathrm{tr}}\right) \quad$ at $x=0$

$k_{2}\left(\underline{\boldsymbol{a}_{\mathrm{t}}} \mathrm{e}^{k_{2} d}-\underline{\boldsymbol{a}}_{\mathrm{tr}} \mathrm{e}^{-k_{2} d}\right)=k_{1} \underline{\boldsymbol{a}}_{\mathrm{t}} \mathrm{e}^{k_{1} d}$ at $x=d$

for their derivatives (velocity), where $d$ is the slab width.

Since the frequency $\omega$ is identical in the two media,

$$
\begin{aligned}
k_{2} & =\frac{V_{\mathrm{A}_{1}}}{V_{\mathrm{A}_{2}}} k_{1} \\
& =\sqrt{\frac{\rho_{2}}{\rho_{1}}} k_{1}=n k_{1}
\end{aligned}
$$

where $V_{\mathrm{A}_{i}}$ and $\rho_{i}$ are the Alfvén velocity and the density in the medium $i$, and $n=n_{2}$ is the (real) refraction index of the medium 2 ( $n_{1}$ being set to 1$)$. In expression $(5 \mathrm{~b})$, we have assumed that the WNM and the CNM are pervaded by the same magnetic field, and therefore $V_{\mathrm{A}_{i}} \propto \rho_{i}^{1 / 2}$. This assumption is based on observational results of magnetic field measurements in the HI interstellar component at scales below $100 \mathrm{pc}$ (Troland \& Heiles 1986; Heiles 1987; Myers et al. 1995) which show that magnetic intensity and gas density are on average uncorrelated in the range 0.1 to $100 \mathrm{~cm}^{-3}$. According both to observations and to biphasic models of the ISM, the density contrast between the CNM and the WNM is typically of the order of 150, and therefore $n \sim 12-13$.

The solutions of systems (3) and (4) are

$$
\begin{aligned}
& \underline{\boldsymbol{a}}_{\mathrm{r}}=(B+\mathrm{i} C) \boldsymbol{a} \\
& \underline{\boldsymbol{a}}_{\mathrm{t}}=(D+\mathrm{i} E) \boldsymbol{a} \\
& \underline{\boldsymbol{a}}_{\mathrm{tr}}=(F+\mathrm{i} G) \boldsymbol{a} \\
& \underline{\boldsymbol{a}}_{\mathrm{t}}=(H+\mathrm{i} J) \boldsymbol{a}
\end{aligned}
$$

where

$$
\begin{aligned}
& B=\left(1-n^{2}\right) K * 2\left(n^{2}+1\right) s^{2} \\
& C=-\left(1-n^{2}\right) K * 4 n s c \\
& D=(n+1) K *\left((n+1)^{2}-(n-1)^{2}\left(c^{2}-s^{2}\right)\right) \\
& E=(n+1) K * 2(n-1)^{2} s c \\
& F=(n-1) K *\left((n+1)^{2}\left(c^{2}-s^{2}\right)-(n-1)^{2}\right) \\
& G=(n-1) K * 2(n+1)^{2} s c \\
& H=4 n K *\left(2 n c * \cos \left(k_{1} d\right)+\left(n^{2}+1\right) s * \sin \left(k_{1} d\right)\right) \\
& J=4 n K *\left(\left(n^{2}+1\right) s * \cos \left(k_{1} d\right)-2 n c * \sin \left(k_{1} d\right)\right)
\end{aligned}
$$

with

$K=\left(8 n^{2}+2\left(n^{2}-1\right)^{2} s^{2}\right)^{-1}$, $s=\sin \left(k_{2} d\right)$ and $c=\cos \left(k_{2} d\right)$.
With these solutions, we compute the different factors entering into play for the transmission and the reflection of energy: (i) the reflected wave amplitude squared $\left(R^{2}\right)$, (ii) the wave amplitude squared $\left(T^{2}\right)$ transmitted to the same medium on the far side of the cloud, and finally (iii) the wave amplitude squared $\left(T I^{2}\right)$ within the cloud.

The reflection factor is obviously given by

$$
\begin{aligned}
R^{2} & =\left|\underline{\boldsymbol{a}}_{\mathrm{r}}\right|^{2} /|\boldsymbol{a}|^{2} \\
& =\left(1+\left(\frac{2 n}{\left(n^{2}-1\right) s}\right)^{2}\right)^{-1}
\end{aligned}
$$

which yields

$R^{2}=0 \quad$ for the resonant modes

$R^{2} \sim 1-\frac{4}{n^{2} s^{2}} \quad$ for the non-resonant modes.

On the other side of the cloud, the transmission factor is given by

$$
\begin{aligned}
T^{2} & =\left|\underline{\boldsymbol{a}_{\mathrm{tt}}}\right|^{2} /|\boldsymbol{a}|^{2} \\
& =\left(1+\frac{\left(n^{2}-1\right)^{2}}{2 n} s^{2}\right)^{-1}
\end{aligned}
$$

from which we see immediately that

$T^{2}=1$ for the resonant modes

$T^{2} \sim \frac{4}{n^{2} s^{2}} \quad$ for the non-resonant modes.

We verify that the relation $R^{2}+T^{2}=1$ holds as is expected for ordinary Fabry-Pérot interferometers.

Finally, we compute the internal coefficient $T I^{2}$. Within the cloud, two plane waves propagate in opposite directions and interfere with each other. Therefore, $T I^{2}$ is given by the following combination of the transmitted wave $\boldsymbol{A}_{\mathrm{t}}$ and the "transreflected" wave $\boldsymbol{A}_{\mathrm{tr}}$ :

$$
\begin{aligned}
T I^{2}= & \left|\underline{\boldsymbol{A}_{\mathrm{t}}}+\underline{\boldsymbol{A}_{\mathrm{tr}}}\right|^{2} /|\underline{\boldsymbol{A}}|^{2} \\
= & \left|(D+\mathrm{i} E) \mathrm{e}^{-i\left(\omega t-k_{2} x\right)}+(F+\mathrm{i} G) \mathrm{e}^{-\mathrm{i}\left(\omega t+k_{2} x\right)}\right|^{2} \\
= & D^{2}+E^{2}+F^{2}+G^{2} \\
& +2(D F+E G) \cos \left(2 k_{2} x\right)+2(G D-E F) \sin \left(2 k_{2} x\right) .
\end{aligned}
$$

Because of the interferences, we obtain a function with spatial dependence. The relevant coefficient to compute is then $T I^{2}$ averaged over the width $d$ of the cloud. The result is

$T I^{2}=D^{2}+E^{2}+F^{2}+G^{2}+\frac{2(D F+E G)}{k_{2} d}|s|$.

The resonant modes correspond to $s \sim 0$. In that situation, if we set apart the particular case of long wavelengths with respect to the slab width $\left(k_{2} d \ll 1\right), T I^{2}$ reduces to

$$
\begin{aligned}
& T I^{2}=\left[D^{2}+E^{2}+F^{2}+G^{2}\right]_{\text {res }} \\
& T I^{2}=\frac{1}{2}\left(1+\frac{1}{n^{2}}\right) .
\end{aligned}
$$


Table 1. Reflection $(R)$, transmission $(T)$ and internal coefficients (TI) in both resonant and non-resonant regimes at the first non vanishing order of $1 / n$.

\begin{tabular}{|c|c|c|}
\cline { 2 - 3 } \multicolumn{1}{c|}{} & At resonance & Out of resonance \\
\hline$R$ & 0 & $1-\frac{2}{n^{2} s^{2}}$ \\
$T$ & 1 & $\frac{2 \sqrt{2}}{n}$ \\
$T I$ & $\frac{\sqrt{2}}{2}$ & $\frac{2}{n}$ \\
\hline
\end{tabular}

In the case of waves with wavelengths far greater than the slab width, the internal coefficient is equal to unity. Such waves set the cloud particles into motion globally, and therefore $T I^{2}=1$ means only that waves with $\lambda_{2} \gg d$ contribute entirely to the cloud bulk motion.

For out of resonance waves, the spatially averaged internal coefficient is

$T I^{2}=\frac{2}{n^{2} s^{2}}\left(1+\frac{1-2 s^{2}}{k_{2} d}+\frac{1}{n^{2}} f(s)\right)$

with $f(s)$ a function of $s=\sin \left(k_{2} d\right)$ only (the full expression of $f(s)$ is given in the caption of Table 2). At this stage, it is important to recall that, as we stressed earlier in this paper, the solutions obtained are those for the motion and velocity amplitudes squared (cf. Eq. (6)). Now, the energy density in waves is $E_{\mathrm{K}}=\frac{1}{2} \rho \delta v^{2}$ and $E_{\mathrm{B}}=\frac{\delta B^{2}}{8 \pi}$ for the kinetic and the magnetic parts respectively. Thus, the ratio of the reflected energy to the incident energy is really $R^{2}$, and the ratio of the transmitted to the incident energy is effectively $T^{2}$ since, in each case, we deal with the same density $\rho_{1}$. However, the ratio of the internal energy to the incident one is not $T I^{2}$, since the density is different in the two media. The actual energy transfer coefficient is

$\frac{E_{\mathrm{K}_{2}}}{E_{\mathrm{K}_{1}}}=\frac{\rho_{2}}{\rho_{1}} \frac{\delta v_{2}^{2}}{\delta v_{1}^{2}}=n^{2} T I^{2}$.

By themselves $R, T$ and $T I$, the square roots derived from Eqs. (7), (9) and (12), can be considered as coefficients defining the rms values of the reflected, transmitted and transferred velocity amplitudes.

For non-resonant wavelengths, they all depend on $s^{2}$. Restricting their expression to the first order in $1 / n$, we then take $\frac{1}{2}$ for the average of $s^{2}$ over a range in cloud sizes. The values for $R, T$ and $T I$ in the different regimes are summarised in Table 1 . The ratio of the internal velocity to the transmitted one, $T I / T$, is identical in both regimes. Moreover, this ratio tends to $\frac{\sqrt{2}}{2}$ with increasing $k$, that is for small wavelengths, independently of resonance (cf. Fig. 2). This means that the energy transferred into the cloud is comparable to the energy transmitted to the other side of the cloud. In other words, the energy in internal motions is of the same order of magnitude as the energy in the bulk motions of the cloud, contrary to what has been claimed in the literature (Elmegreen 1985). This significant difference, sketched in Fig. 2, is exclusively due to the inclusion of interferences within the cloud which have not been previously taken into account.
Table 2. rms ratios of the amplitudes and the energy densities in both media (cloud and inter-cloud) computed for resonant and nonresonant wavelengths. The expressions given for the resonant case are exact, whereas expressions given for non resonant wavelengths are second order approximates (with $g\left(k_{2}, s\right)=\left(1+\frac{1-2 s^{2}}{k_{2} d}+\frac{1}{n^{2}} f(s)\right)$ where $f(s)=3(1-3|s|)-2|s|^{3}-\frac{4}{s^{2}}(1+|s|), s=\sin \left(k_{2} d\right)$ where $d$ is the width of the cloud; $\left.n=\sqrt{\rho_{2} / \rho_{1}}\right)$.

\begin{tabular}{ccc}
\hline \hline $\begin{array}{c}\mathrm{rms} \\
\text { ratio }\end{array}$ & $\begin{array}{c}\text { At } \\
\text { resonance }\end{array}$ & $\begin{array}{c}\text { Out of } \\
\text { resonance }\end{array}$ \\
\hline$\frac{\delta v_{2}^{2}}{\delta v_{1}^{2}}$ & $\frac{1}{2}\left(1+\frac{1}{n^{2}}\right)$ & $\frac{2}{n^{2} s^{2}} g\left(k_{2}, s\right)$ \\
$\frac{E_{\mathrm{K}_{2}}}{E_{\mathrm{K}_{1}}}$ & $\frac{1}{2}\left(n^{2}+1\right)$ & $\frac{2}{s^{2}} g\left(k_{2}, s\right)$ \\
$\frac{E_{\mathrm{B}_{2}}}{E_{\mathrm{B}_{1}}}$ & $\frac{1}{2}\left(n^{2}+1\right)$ & $\frac{2}{s^{2}} g\left(k_{2}, s\right)$ \\
\hline
\end{tabular}

\subsection{Internal energy}

A far more interesting consequence of the inclusion of internal interferences is the fate of the energy densities within the cloud. It is straightforward to see that the density of energy carried by the waves is equally distributed between kinetic and magnetic degrees of freedom. Let us then compute just the kinetic part of it.

For each frequency, the kinetic energy density in media (1) and (2) writes $E_{\mathrm{K}_{i}}=\frac{1}{2} \rho_{i} \delta v_{i}^{2}$, where $i \in\{1,2\}$. As we wrote earlier, the ratio of the kinetic energy densities is

$\frac{E_{\mathrm{K}_{2}}}{E_{\mathrm{K}_{1}}}=\frac{\rho_{2}}{\rho_{1}} \frac{\delta v_{2}^{2}}{\delta v_{1}^{2}}=n^{2} T I^{2}$

where $T I^{2}$ is given by Eqs. (12)-(14). As we can see, in the resonant regime, the internal energy density of each type (kinetic and magnetic) due to Alfvén waves amounts to $n^{2} / 2$ times the external energy density. Thus, the enhancement in internal energy due to the sharp discontinuity in the matter density is colossal since, for typical values of the ISM, $n^{2}=\rho_{2} / \rho_{1}$ reaches $10^{2}$. Then, even in the non-resonant regime, if we take again $\left\langle s^{2}\right\rangle=0.5$, the density of energy in the cloud is four times bigger than the energy density carried by the incident waves. Table 2 summarizes the values taken by the energy ratio in the different modes.

These are the results for the energy transmission per frequency $\omega$. However, for the question of stabilising efficiency, we need to know what happens when the density discontinuity is attacked by a spectrum of waves. Will the effect of resonances be smeared away when summing up the contribution of a whole spectrum of waves? As we have seen above, even in a non-resonant regime, the internal energy density carried by a wave of a given wavelength is greater than the incident energy density. Therefore, we expect the effect of resonances to be diminished, but not completely destroyed.

Consider now a group of clouds evolving in the potential well of a host galaxy and connected to each other by magnetic field lines. Various effects such as supernovæ explosions and orbital motions of the clouds contribute to the generation of a spectrum of hydromagnetic waves (e.g. Falgarone \& Puget 1986). In such a situation, magnetosonic waves are much more 


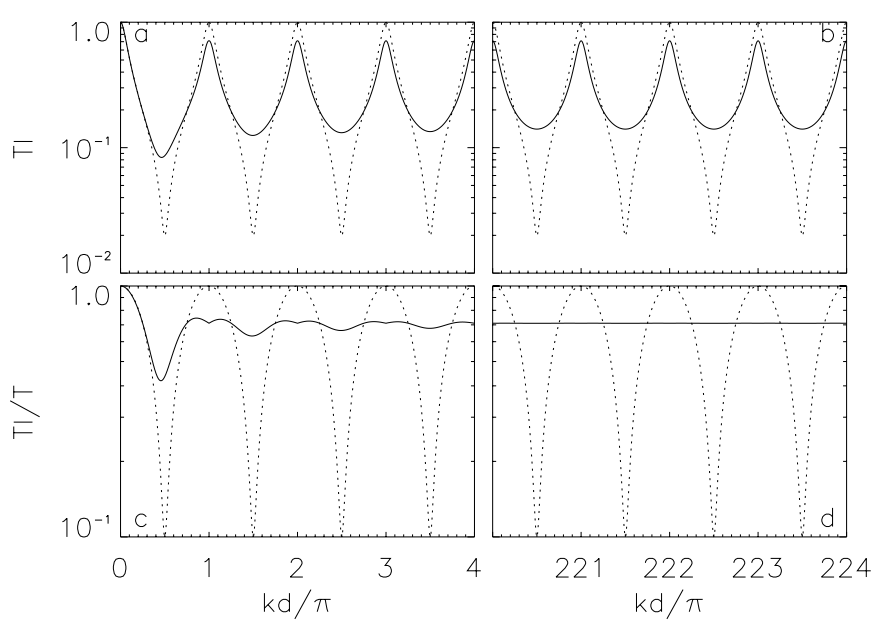

Fig. 2. Transfer coefficient (upper panels) and its ratio to the transmission coefficient (lower panels) as functions of wave number $k$. Drawn are both the expression which take internal interferences into account (plain line) and the expression which does not (dotted line), for long wavelengths (panels a) and c)) and for short wavelengths (panels b) and d)). For illustration, the ratio of cloud density to intercloud density is taken to be $n=10$. The most important feature is the limit of $\frac{\sqrt{2}}{2}$ reached by the ratio of internal to bulk motions, independently of the regime (panel d)).

affected by damping processes than Alfvén waves (Zweibel \& Josafatsson 1983; McKee \& Zweibel 1995). Therefore, at the boundaries of each cloud, we can focus our attention on the alfvénic part of the wave spectrum only.

Considering that energy is roughly equally distributed among the different degrees of freedom, the amplitude of the velocity perturbations in the intercloud medium is of the order of the Alfvén speed at an injection scale $L=\frac{2 \pi}{K}$ comparable to the mean distance between the clouds. At the external boundary of a cloud, the Alfvén wave spectrum can be expressed as

$\delta v_{1}^{2}(k)=V_{\mathrm{A}_{1}}^{2}\left(\frac{k}{K}\right)^{-\alpha}$

where $V_{\mathrm{A}_{1}}$ is the Alfvén speed in the medium 1. At the injection scale $L$, we have $k=K$ and $\delta v_{1}^{2}(K)=V_{\mathrm{A}_{1}}^{2}$ which provides the normalisation of the spectrum. The wave spectrum inside the clouds is just the one above multiplied by the transfer coefficient derived in (12):

$\delta v_{2}^{2}(k)=V_{\mathrm{A}_{1}}^{2} T I^{2}(k)\left(\frac{k}{K}\right)^{-\alpha}$,

and the additional energy density due to this transfer of waves is therefore

$E_{\mathrm{K}_{2}}=\frac{1}{2} \rho_{2} V_{\mathrm{A}_{1}}^{2} d \int_{k_{\text {inf }}}^{k_{\text {sup }}} T I^{2}(k)\left(\frac{k}{K}\right)^{-\alpha} \mathrm{d} k$
$E_{\mathrm{K}_{2}}=n^{2} \frac{B^{2}}{8 \pi} d \int_{k_{\text {inf }}}^{k_{\text {sup }}} T I^{2}(k)\left(\frac{k}{K}\right)^{-\alpha} \mathrm{d} k$

where the last line comes from the definition of the Alfvén velocity, $V_{\mathrm{A}_{1}}=\frac{B}{\sqrt{4 \pi \rho_{1}}}$. The lower limit in wave number space, $k_{\text {inf }}$, corresponds to the first mode which contributes to the support of the slab. By choosing this lower cut-off, we do not include the bulk motion of the slab induced by the waves. We

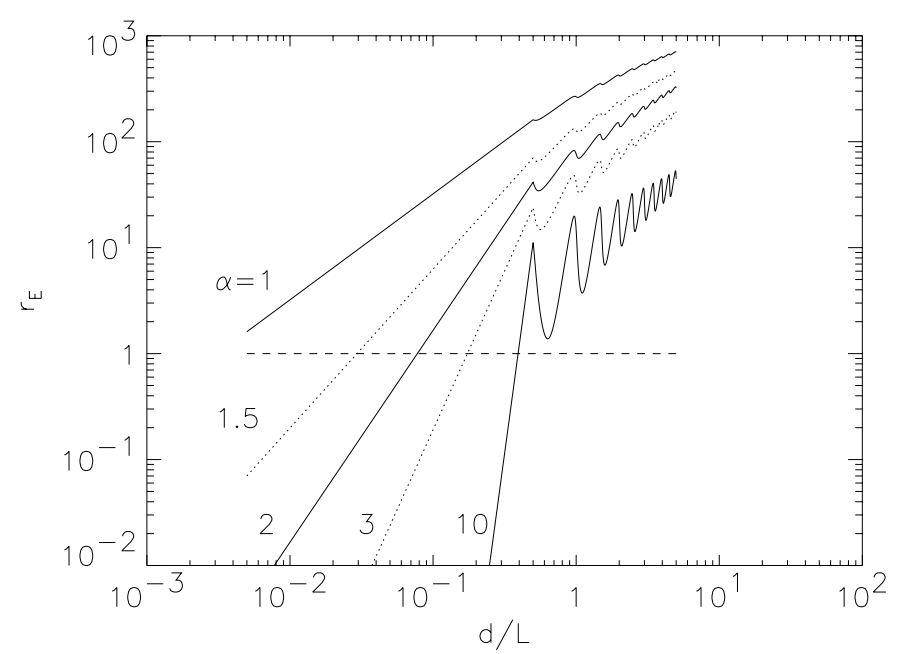

Fig. 3. Ratio $r_{\mathrm{E}}$ of the transmitted kinetic energy in Alfvén waves to the energy contained in the background magnetic field $B$ as a function of the ratio of the slab width to the mean distance between slabs $d / L$. Several values of the wave spectral index $\alpha$ were adopted. From top to bottom, $\alpha=1,1.5,2,3,10$. The horizontal line indicates where the transferred turbulent energy becomes comparable to the energy provided by the background magnetic field. The oscillations visible for $d>0.5 L$ correspond to the domination of coherent (resonant modes) or incoherent (non-resonant modes) summation (peaks and dips respectively). Calculations were performed with $n \simeq 12.25$.

take into account only the internal motions which are relevant for the stability of the slab. If the mean distance $L$ between slabs is larger than twice the slab width, the first mode opposing external compression is the one whose wavelength is twice the slab width $d$, i.e. $k_{\text {inf }} \simeq \pi / d$ which, for the typical size of a CNM cloud ( $d \sim 1.5 \mathrm{pc}$, McKee \& Ostriker 1977), gives

$k_{\mathrm{inf}} \simeq 6.79 \times 10^{-19} \mathrm{~cm}^{-1}$.

Otherwise, the lower limit $k_{\text {inf }}$ is set by the lowest excited mode in the external medium, $K=2 \pi / L$. Therefore $k_{\text {inf }} \simeq$ $\max \left\{\frac{\pi}{d}, \frac{2 \pi}{L}\right\}$.

The upper limit is set by the wave number $k_{\text {sup }}=\frac{2 x_{i} v_{\text {in }}}{V_{\mathrm{A}_{2}}}$ for which the wave frequency $\omega$ becomes comparable to the ion-neutral collision frequency $v_{\text {in }}\left(x_{\mathrm{i}}\right.$ is the ionisation fraction and $V_{\mathrm{A}_{2}}$ is the Alfvén velocity inside the cloud). Using Eq. (7) of McIvor (1977) for the ion-neutral collision frequency, and with typical values of density and ionisation fraction for the CNM $\left(n \sim 61 \mathrm{~cm}^{-3}, x_{\mathrm{i}} \sim 3 \times 10^{-4}\right.$, see e.g. Wolfire et al. 1995), we find

$k_{\text {sup }} \simeq 4.15 \times 10^{-17} \mathrm{~cm}^{-1}$

for a magnetic field strength of $5 \mu \mathrm{G}$.

We integrated Eq. (20) numerically for a set of values of the spectral index $\alpha$. Results are shown in Fig. (3) in terms of $r_{\mathrm{E}}=E_{\mathrm{K}_{2}} /\left(B^{2} / 8 \pi\right)$, which is the ratio of the internal kinetic energy in Alfvén waves to the energy in the background field $B$, as a function of the ratio of the slab width $d$ to the mean slab separation $L$. Intuitively enough, steep wave spectra feed less wave pressure into the cloud than shallow spectra. Nevertheless, even for an extreme spectral index such as $\alpha=10$, the total internal Alfvén wave pressure becomes comparable to the pressure of 
the background magnetic field when the mean separation between slabs is smaller than roughly 2.5 times the slab width. More strikingly perhaps, for more reasonable spectral indices ( $\alpha \simeq 2-3$, say), magnetic support provided by internal motions can be an order of magnitude larger than support provided by the mean field when the two length scales become comparable. Thus, as we guessed above, resonant wave energy transfer is not washed out when a full spectrum of waves is considered.

\section{Discussion}

As we have seen, in the stationary case, the density of energy due to the injection of a spectrum of shear Alfvén waves into the cloud represents a non negligible fraction of the total energy available within the cloud. In some cases, its contribution is even dominant over the part provided by the static background magnetic field. This energy comes into play in the virial equilibrium of the cloud in the form of non-thermal pressure, providing additional support against self gravity and external pressure of the reflected waves.

The results presented here were derived for a very simplified case. First of all, the assumed geometry is very peculiar and the cloud boundaries are extremely sharp. In reality, the situation is more complicated since the clouds assume complex shapes. Moreover, the cloud boundary is probably less well defined, although under the action of thermal instability the density discontinuity is actually sharp. The effects of a progressive increase in density, instead of an abrupt step, are difficult to estimate. The refraction index $n=\left(\frac{\rho_{2}}{\rho_{1}}\right)^{1 / 2}$ will be in that case an increasing function of the distance within the boundary layer before reaching a plateau inside of the cloud. Actually, according to the so-called ray equation $\left(\frac{\mathrm{d}(n \boldsymbol{u})}{\mathrm{d} s}=\boldsymbol{\nabla} n\right.$ where $\boldsymbol{u}$ is the unity vector aligned with $\boldsymbol{k}$ and $s$ the curvilinear abscissa along the wave path), a gradient in the $n$ index will bend the trajectory of the waves in the direction of increasing $n$, i.e. toward the interior of the cloud. Since the waves are originally propagating along the mean magnetic field lines, the bending of their path will result in the coupling of Alfvén modes to magnetosonic modes. The latter are known to be more dissipative than shear Alfvén waves, and the energy transmission will thus be diminished. However, as long as the thickness of the boundary layer remains negligible with respect to the cloud size, the situation is likely not to differ very much from what has been presented in the previous sections.

The problem of finite boundary size is of particular interest within the context of molecular clouds. These are generally self gravitating systems, not necessarily in thermal pressure equilibrium with their surroundings, and the discontinuity between molecular clouds and the surrounding medium is smoothed by gravity. Furthermore, molecular clouds are also denser than the atomic media studied in this paper. The magnetic field strength cannot be considered anymore independent of density (as we have assumed in Sect. 2.1). Indeed, as detailed calculations of Mouschovias (1976) show for thermally supported clouds, the mean magnetic field strength $B$ scales with the average gas density $\rho$ as

$B \propto \rho^{\kappa}$ where $1 / 3 \leq \kappa \leq 1 / 2$, and for an isotropically contracting cloud, $\kappa=2 / 3$. Nevertheless, if the boundary layer is sufficiently thin, the energy injection mechanism explored in this study should remain valid even in the case of molecular clouds connected by a magnetic field, provided that the refraction index is modified as to match the magnetic strength scaling with density. This is easily done by replacing $n$ by $n^{\prime}=V_{\mathrm{A}_{1}} / V_{\mathrm{A}_{2}}$ as in the definition of the refraction index in Eq. (5a). With the latter modification, our study applied to molecular clouds corroborates the results found by Falgarone $\&$ Puget (1986). For the conditions adopted in their paper, we obtain a refraction index $n^{\prime} \simeq 8$ and a maximum wave number $k_{\text {sup }} \simeq 1.76 \times 10^{-17} \mathrm{~cm}^{-1}$. In their study, the cloud width is $d=1.5 \mathrm{pc}$ and the mean distance between clouds is $L=8 \mathrm{pc}$. With these values, the ratio of the injected energy to the energy in the background magnetic field amounts to

$r_{\mathrm{E}} \simeq 0.20-3.65$

for a spectral index $\alpha=2-4$. Therefore, the energy in the random internal magnetic field and the energy associated with the mean field are of the same order of magnitude, which is precisely what Falgarone \& Puget obtained. However, in their paper, following a study of Clifford \& Elmegreen (1983), magnetic field lines bending at the cloud boundary gives birth to a spectrum of waves directly within the clouds while the external field remains essentially unperturbed. On the contrary, we assumed that the wave spectrum is generated outside of the clouds and we examined its transfer into the clouds. Our study thus provides an alternate picture of the mechanism of energy transfer mediated by the magnetic field.

Another restriction of the model presented here is the assumption of steady state. As we have said in Sect. 2.1, the steady state corresponds to an infinite number of internal reflections. Now, when a transverse wave hits the outer surface of a cloud, its first effect is to compress it. Thus, the cloud begins to shrink, increasing in density and in temperature. Depending on its cooling efficiency, the cloud may become gravitationally unstable in the direction parallel to the mean magnetic field, and collapse before the steady state is reached. However, the compression of the cloud would propagate at the cloud internal sound speed $C_{\mathrm{s}}$, which is roughly $C_{\mathrm{s}} \simeq 0.61 \mathrm{~km} \mathrm{~s}^{-1}$ for $T=45$ Kelvins, whereas waves propagate at the internal Alfvén speed $V_{\mathrm{A}_{2}} \simeq 1.4 \mathrm{~km} \mathrm{~s}^{-1}$. Thus, the transfer of energy in that case could be efficient enough and the internal turbulent energy could be rapidly sufficient to counteract the external wave pressure. In that case, mhd-turbulent support would not be limited to the case of outwardly propagating Alfvén waves, contrary to what has been suggested previously (Shu et al. 1987).

We are currently undertaking simulations of a more realistic case where the transition layer between the slabs and the intercloud medium is properly described. The spectrum of waves in the external medium is generated by an ensemble of slabs in relative motions which allows to test the spectral index $\alpha$ and the normalisation of the spectrum. Results will be presented in a forthcoming article. 


\section{Conclusion}

We examined, in the simplified case of slab geometry and steady state, the transfer of energy through shear Alfvén waves from large scales to small scales in the neutral ISM. At large scales, energy is injected by bulk motions of cold clouds connected by a magnetic field and virialised in the gravitational potential well of the Galaxy. These motions generate a spectrum of hydromagnetic waves which propagate along the field lines. In this work, we concentrated exclusively on the efficiency of energy transfer in a thermally bistable magnetised medium where clouds are in large density contrast with their surroundings.

At the cloud boundaries, the waves are partially reflected and partially transferred into the cloud. Taking explicitly into account internal interferences, we first re-derived the reflection, transmission and transfer coefficients. Our results for the transfer coefficient differ from those available in the literature. Given the correction, we have shown that the energy in internal motions is of the same order of magnitude as the energy in bulk motions, contrary to what has been claimed earlier.

Moreover, resonant interactions of back and forth traveling waves within the clouds drive internal motions and enhance considerably the energy transfer. Depending on the geometry and on the spectral index of the wave spectrum, the energy of internal motions integrated over the whole spectrum contributes significantly to the global energy budget of the clouds. In some cases, energy provided by the injected Alfvén waves can be as large as ten times the energy of the background magnetic field. This makes the injected waves and their interferences a potentially important mean to establish pressure equilibrium between the clouds and the intercloud medium.

Provided slight changes in the definition of the refraction index, the formalism developed in this study could hold equally for the case of molecular clouds, as long as the thickness of the transition layer between the intercloud medium and the molecular clouds is small with respect to the cloud size.

Our study is clearly far from providing a self-consistent model of the cloud hierarchy in the ISM. We showed nevertheless that energy transfer via Alfvén waves represents a potentially important ingredient for such a model.

Acknowledgements. We thank an anonymous referee for pointing to us some overlooked references and for useful comments which helped us to improve the quality of this manuscript. M.L. also thanks gratefully for their hospitality members of the Astrophysics Department, University of Oxford, where part of this work has been done, supported by a Marie Curie Fellowship of the
European Community "Human Potential" programme, contract number HPTM-CT-2000-151.

\section{References}

Arons, J., \& Max, C. E. 1975, ApJ, 196, L77

Ballesteros-Paredes, J., Vázquez-Semadeni, E., \& Scalo, J. 1999, ApJ, 515,286

Carlberg, R. G., \& Pudritz, R. E. 1990, MNRAS, 247, 353

Caselli, P., \& Myers, P. C. 1995, ApJ, 446, 665

Clifford, P., \& Elmegreen, B. G. 1983, MNRAS, 202, 629

Coker, R. F., Rae, J. G. L., \& Hartquist, T. W. 2000, A\&A, 360, 290

Elmegreen, B. G. 1985, ApJ, 299, 196

Elmegreen, B. G. 1999, ApJ, 527, 266

Falgarone, E., \& Phillips, T. G. 1991, in Fragmentation of Molecular Clouds and Star Formation, IAU Symp., 147, 119

Falgarone, E., \& Puget, J. L. 1986, A\&A, 162, 235

Falgarone, E., \& Puget, J. L. 1988, in NATO ASIC Proc. 232, Galactic and Extragalactic Star Formation, 195

Falle, S. A. E. G., \& Hartquist, T. W. 2002, MNRAS, 329, 195

Field, G. B., Goldsmith, D. W., \& Habing, H. J. 1969, ApJ, 155, L149

Gammie, C. F. \& Ostriker, E. C. 1996, ApJ, 466, 814

Heiles, C. 1987, in Interstellar Processes, ASSL, 134, 171

Hennebelle, P., \& Pérault, M. 1999, A\&A, 351, 309

Hennebelle, P., \& Pérault, M. 2000, A\&A, 359, 1124

Koyama, H., \& Inutsuka, S. 2000, ApJ, 532, 980

Koyama, H., \& Inutsuka, S. 2002, ApJ, 564, L97

Kritsuk, A. G., \& Norman, M. L. 2002, ApJ, 569, L127

Larson, R. B. 1981, MNRAS, 194, 809

Martin, C. E., Heyvaerts, J., \& Priest, E. R. 1997, A\&A, 326, 1176

McIvor, I. 1977, MNRAS, 178, 85

McKee, C. F. 1995, in The Physics of the Interstellar Medium and Intergalactic Medium, ASP Conf. Ser., 80, 292

McKee, C. F., \& Ostriker, J. P. 1977, ApJ, 218, 148

McKee, C. F., \& Zweibel, E. G. 1995, ApJ, 440, 686

Mouschovias, T. C. 1976, ApJ, 207, 141

Myers, P. C., Goodman, A. A., Gusten, R., \& Heiles, C. 1995, ApJ, 442, 177

Pérault, M., Falgarone, E., \& Puget, J. L. 1986, A\&A, 157, 139

Sánchez-Salcedo, F. J., Vázquez-Semadeni, E., \& Gazol, A. 2002, ApJ, 577, 768

Scalo, J. M. 1987, in Interstellar Processes, ASSL, 134, 349

Shu, F. H., Adams, F. C., \& Lizano, S. 1987, ARA\&A, 25, 23

Solomon, P. M., Rivolo, A. R., Barrett, J., \& Yahil, A. 1987, ApJ, 319, 730

Troland, T. H., \& Heiles, C. 1986, ApJ, 301, 339

Vázquez-Semadeni, E., Gazol, A., \& Scalo, J. 2000, ApJ, 540, 271

Wolfire, M. G., Hollenbach, D., McKee, C. F., Tielens, A. G. G. M., \& Bakes, E. L. O. 1995, ApJ, 443, 152

Zweibel, E. G., \& Josafatsson, K. 1983, ApJ, 270, 511

Zweibel, E. G., \& McKee, C. F. 1995, ApJ, 439, 779 